\title{
An application of the augmented $\varepsilon$-constraint method to design a municipal sorted waste collection system
}

\author{
Diego Gabriel Rossit ${ }^{a^{*}}$, Fernando Abel Tohméb, Mariano Frutos ${ }^{\mathrm{a}}$ and Diego Ricardo Broz ${ }^{\mathrm{c}}$
}

${ }^{a}$ IIESS, Department of Engineering, Universidad Nacional del Sur (UNS)-CONICET, Argentina

${ }^{b} I N M A B B$, Department of Economics, Universidad Nacional del Sur (UNS)-CONICET, Argentina

${ }^{c}$ Faculty of Forest Sciences, Universidad Nacional de Misiones and CONICET, Argentina

\section{H R O N I C L E \\ A B S T R A C T}

Article history:

Received Febryary 16, 2017

Received in revised format:

February 22, 2017

Accepted March 28, 2017

Available online

March 282017

Keywords:

Municipal solid waste

Sustainability

Multi-objective capacitated

facility location problem

\begin{abstract}
The separation at the source of Municipal Solid Waste (MSW) is an initiative that facilitates the subsequent recycling work and contributes to palliate the negative impacts of the traditional unsorted collection system. This paper presents a multi-objective integer linear programming model of the determination of the optimal location of assorted waste bins in an urban area. We consider, jointly, the objectives of minimizing the investment cost and the average distance from the dwellings to the bins. The model was applied in simulated instances of an Argentinian medium-size city, contributing to the transition from the current door-to-door based system to a community bins system. To solve this problem, we apply both the weighting method, which has been used to solve similar problems in the literature, and a novel version of the augmented $\varepsilon$-constraint method (AUGMECON2). The results over simulated scenarios show that, in general, AUGMECON2 has a better performance, yielding a larger number of efficient solutions at lower computation times.
\end{abstract}

\section{Introduction}

The rise in world population and the associated increase in urbanization have put pressure on the management of Municipal Solid Waste (MSW), which is regularly associated with various environmental, social and economic problems (Poletto et al., 2016; Troschinetz \& Mihelcic, 2008). These problems intensify in developing countries, due mainly to budgetary and technological restrictions. In order to reduce these impacts and provide an efficient MSW management, there are some activities that must be adequately performed: collection, transformation and disposal (Ghiani et al., 2014b). The potential contribution of Operations Research to run more efficiently these operations is enormous. Ghiani et al. (2014a) presented an extensive and thorough review of the state-of-the-art literature of the application of Operations Research to MSW management.

* Corresponding author.

E-mail address: diego.rossit@uns.edu.ar (D. G. Rossit)

C 2017 Growing Science Ltd. All rights reserved. doi: $10.5267 /$ j.dsl.2017.3.001 
Considering the complexity of handling different flows of garbage in opposition to the more homogenous waste streams resulting from industrial or agricultural activities, one of the strategies that can contribute to a healthier MSW management system is source separation (Hui et al., 2006; Zhuang et al., 2008). Usually, the implementation of a source separation MSW system involves locating facilities in proper places, fulfilling some desired criteria. In the literature this kind of problem is known as a Facility Location Problem (FLP): given a set of potential locations of facilities and a set of demand points the objective is to localize facilities in a way that the total cost of opening and allocating customers is minimized (Salemi, 2016). The problem of locating capacitated facilities, so-called the Capacitated Facility Location Problem (CFLP), in the context of solid waste management has been extensively studied in the literature; reflecting not only the rise in social and environmental awareness on MSW management but also the complexity of locating semi-obnoxious facilities (Crespo \& Paredes, 2009). The problem has usually been addressed considering just one objective; for instance, the minimization of the total number of facilities (Ghiani et al., 2012) or the sum of fixed and variable costs (Badran \& El-Haggar, 2006; Ferri et al., 2015, Pérez-Salazar et al., 2015). On the other hand, other authors dealt with more than one criterion (Coutinho-Rodrigues et al., 2012; Erkut et al., 2008; Rossit et al., 2015, Tralhão et al., 2010). Some authors have solved the CFLP with a multi-objective perspective through the weighting method (Coutinho-Rodrigues et al., 2012; Rossit et al., 2015; Tralhão et al., 2010). Although this is useful to generate a set of non-dominated solutions, it is known that this method presents important disadvantages for the generation of Pareto optimal solutions. This will be discussed in detail in Section 2. In the literature we can also find stochastic approaches to the problem (Toso \& Alem, 2014).

\subsection{The Argentinian MSW system and case of application}

All along this highly urbanized developing country, the mishandling of garbage is commonplace. According to the Ministry of Environment and Sustainable Development of Argentina (MADS, 2016) only $61 \%$ of the population has a correct disposition of municipal waste, i.e., has adequate landfills. Moreover, only $37 \%$ of the municipalities of Argentina perform some sort of waste classification. However, most of the cities that separate different kinds of waste, perform an unsorted collection of garbage and then classify it in special facilities. Only a handful of localities implement source separation collection. Among them, some large cities from the central part of the country like Buenos Aires (Bonomo et al., 2012) and Rosario (Gandara \& Guerrero, 2013) have started implementing source separation systems through community containers. However, most of the municipalities that implement source separation, e.g., the northern city of Salta (Tufró et al., 2012), keep running a door-to-door system of individual household waste receptacles. In this system, the classification is performed by setting different collection frequencies for the different kinds of garbage. Therefore, the logistic complexity of the routing schedule is rather high.

Some years ago, the government of the southern city Bahía Blanca, which consitiutes the case of application of our model, being aware that the landfill is about to collapse, has also tried to implement a source separation system maintaining the door-to-door basis. However, this methodology was abandoned because of lack of political determination. Bahía Blanca is a medium city with approximately 300,000 inhabitants, which makes it the biggest city in the south of Argentina, and is an important industrial and intermodal transport center for the Argentinian economy. As mentioned, waste collection is done in a door-to-door (or house-to-house) basis, where collectors visit each individual dwelling to collect garbage. As in many urban conglomerations, local authorities are responsible for handling MSW from the initial point of generation to the final disposal or treatment (Pradhan et al., 2012). In part because of the remarkably high Argentinian logistic costs (Broz et al., 2016), including the high salaries that are paid to members of the transport's trade union in Argentina (Benes \& Milmanda, 2012), the MSW system consumes about 30\% of the municipal government budget. Besides that, it is highly inefficient in accomplishing this task. This is quite common in developing countries (Hoornweg \& Bhada-Tata, 2012). Most of the collected waste ends in the same landfill, which, to make 
matters worse, is close to reach its full capacity. The climate of the city, which is rather cold and dry, since Bahía Blanca is located at the gates of the arid Patagonian desert, does not facilitate natural decomposition in the landfill. Only a tiny portion of the garbage is collected from six "clean points", i.e., areas where different containers are located to receive different flows of waste. This classified garbage is sent to a waste treatment plant for recycling and further classification. But the impact of six special sites for source separation in a city of 300,000 people is relatively small. Furthermore, the location of these containers has not been optimized to make their use simpler for the citizens. These "clean points", which are usually located in busy places, are mainly for educational and promotional purposes rather than part of a real large-scale collection system.

It is highly relevant, thus, to seek sustainable solutions for the improvement of the MSW management. These policies should solve two major problems: reduce the huge amounts of resources that are invested on MSW and improve the sustainability of the system. The idea of this paper is to present a model that can be useful for decision-makers in order to implement a source-separated system that can support a sustainable treatment of garbage. Based on the fact that using community containers instead of the dwelling's individual receptacles can enhance efficiency and reduce environmental problems (Bonomo et al., 2012), we propose a migration from the current door-to-door system to a community bins system, where users bring their garbage to community containers placed at fixed points in a neighborhood or locale. In addition to this, we propose that the community bins support garbage's classification. This means that in the collection sites, people should find different containers for different kinds of waste. The extension of a community containers can help both to reduce the logistic complexity of the problem, because fewer locations have to be visited by the trucks and to promote recycling and reutilization, because of the physical separation of the different flows of garbage. However, the distribution of containers has to be cleverly defined since it should take into account the necessities of citizens in terms of both amount of waste produced and distance from dwellings (Parrot et al., 2009).

The article is structured as follows: in the Section 2 we present an Integer Programming model and two methods that we will use to solve it, in Section 3 we present the results of the application of the model in simulated instances of the urban structure of Bahía Blanca and in Section 4 we outline some conclusions.

\section{Material and methods}

An Integer Programming (IP) model allows obtaining the optimal location of collection sites, i.e., an area where waste bins can be installed and the containers to be placed there. In order to proceed with the installation of the containers in a specific collection site, the site has to be opened to that effect. In our model, two objectives are pursued: the minimization of the average distance from the dwellings to the respective assigned containers and the minimization of investment costs, including those of opening collection sites as well as the expenses of installing containers. The model determines the optimal allocation of bins to open collection sites as well as the assignment of dwellings to the collection sites such a way that the demands of different types of waste are satisfied. The containers can be of diverse types, with differences in capacity and installation space. We also take into account that there is a maximum admissible distance between a dwelling and its assigned container. We call it the threshold distance. This concept has been used regularly in the bibliography (Coutinho-Rodrigues et al., 2012; Di Felice, 2014a; Ghiani et al. 2014b; Ghiani et al., 2012; Rossit et al., 2015; Tralhão et al., 2010) and is relevant in the design of a collection system accessible to users. The sets, parameters and variables used in this model are as follows,

\begin{tabular}{ll}
\hline Sets & \\
\hline$I$ & Set of dwellings. \\
$J$ & Set of candidate collection sites. \\
$M$ & Set of types of waste. \\
$H$ & Set of class of containers. \\
\hline
\end{tabular}




\begin{tabular}{|c|c|}
\hline \multicolumn{2}{|l|}{ Parameters } \\
\hline$d_{i, j}$ & Distance in meters between the dwelling $i$ to the collection site $j$. \\
\hline$c$ & Cost in monetary units (m.u.) of opening a candidate collection site. \\
\hline$q_{h}$ & Cost in m.u. of installing a bin of class $h$. \\
\hline$b_{i, m}$ & Quantity in liters of waste of type $m$ produced by dwelling $i$. \\
\hline $\operatorname{cap}_{h}$ & Capacity of bin of type $h$ in liters. \\
\hline$L$ & Maximum space in $\mathrm{m}^{2}$ in a collection site. \\
\hline$l_{h}$ & Space in $\mathrm{m}^{2}$ needed by a bin of class $h$. \\
\hline$d_{\text {máx }}$ & $\begin{array}{l}\text { Threshold distance between a dwelling and its assigned collection site } \\
\text { in meters. }\end{array}$ \\
\hline \multicolumn{2}{|l|}{ Binary Variables } \\
\hline$x_{i, j}$ & 1 if the dwelling $i$ is assigned to the collection site $j, 0$ otherwise. \\
\hline$y_{j}$ & 1 if the candidate collection site $j$ is open, 0 otherwise. \\
\hline \multicolumn{2}{|l|}{ Integer Variables } \\
\hline$k_{h, m, j}$ & $\begin{array}{l}\text { Number of bins of class } h \text { and waste type } m \text { installed in the collection } \\
\text { site } j \text {. }\end{array}$ \\
\hline
\end{tabular}

The IP model determining the collection sites to be opened and the location of the containers is presented in Eqs. (1-8).

$\min \left\{f_{c}, f_{d}\right\},\left\{\begin{array}{c}f_{c}=\sum_{\substack{j \in J \\ m \in M \\ h \in H}}\left(k_{h, m, j} \cdot q_{h}\right)+\sum_{j \in J}\left(y_{j} \cdot c\right) \\ f_{d}=\frac{\sum_{j \in I}\left(d_{i, j} \cdot x_{i, j}\right)}{I}\end{array}\right.$

subject to:

$$
\begin{aligned}
& \sum_{j \in J} x_{i, j}=1, \forall i \in I \\
& \sum_{h \in H} k_{h, m, j} \cdot l_{h} \leq L \cdot y_{j}, \forall j \in J \\
& \sum_{i \in I}\left(b_{i, m} \cdot x_{i, j}\right) \leq \sum_{h \in H}\left(c a_{h} \cdot k_{h, m, j}\right), \forall j \in J, m \in M \\
& x_{i, j} \cdot d_{i, j} \leq d_{m a x}, \forall i \in I, j \in J \\
& x_{i, j} \in[0,1], \forall i \in I, j \in J \\
& y_{j} \in[0,1], \forall j \in J \\
& k_{h, m, j} \in Z^{+}, \forall h \in H, m \in M, j \in J
\end{aligned}
$$


Eq. (1) describes the objectives: the minimization of both the investment cost $\left(f_{c}\right)$ and the average distance from dwellings to the respective assigned collection sites $\left(f_{d}\right)$. Eq. (2) establishes that a dwelling can be only assigned to one collection site. Inequality (3) ensures that the quantity of bins installed in an open collection site does not exceed the maximum space capacity of the place. Inequality (4) states that the amount of each type of waste assigned to an open collection site does not exceed the capacity of the installed bins for that type of waste. Eq. (5) states that a dwelling cannot be assigned to a container that is beyond the threshold distance. This inequality can be eliminated if we leave undefined the values of variables $x_{i, j}$ for combinations $(i, j)$ such that $d_{i, j}>d_{\max }$. Conditions (6) and (7) indicate the binary nature of the variables. Finally, (8) states that the variable is a non-negative integer.

There exists a clear conflict between the two objectives of the model. In spite of the undesirable high investment cost of establishing numerous collection sites and bins this ensures a short average distance between dwellings and their assigned bins. This also increase the time needed to collect garbage due to a larger number of points to visit (Di Felice, 2014b). Consequently, while a small quantity of open collection sites and containers is less costly, it imposes longer waste carrying distances to the users which can negatively affect the accessibility to the system (Parrot et al., 2009). Di Felice (2014b) also stated that this would imply the location of large concentration of containers in a collection point what affecting the aesthetics of the area and, therefore, the commercial value of the surroundings dwellings.

\subsection{Multi-objective approach}

Multi-objective optimization in MSW management can offer a comprehensive view to decision makers since solutions are not only guided by the goal of minimizing costs (Mavrotas et al., 2015). Hwang and Masud (1979) classify multi-objective problems in three categories, based on the phase in which the decision maker becomes involved: the a priori methods, the interactive methods and the a posteriori or generation methods. In the case of a priori methods, the decision maker expresses his preferences before engaging in a calculation process. In interactive methods, deliberations with the decision-maker are alternated with optimization processes. The decision maker progressively drives the search with his answers towards the most preferred solution. Finally, in a posteriori methods, a phase in which efficient solutions of the problem are generated (all of them or a proper sample) is followed by one in which the decision maker selects the most preferred one. Generation methods require large computational efforts because the calculation of efficient solutions is usually a time consuming process. However, they become suitable when the decision maker is not initially available since he has to participate only in the last phase of the process. An additional advantage of generation methods stems from the fact that the decision maker gets a thorough panorama of the alternatives before making the final decision, reinforcing his confidence (Mavrotas, 2009).

In Multi-Objective Mathematical Programming (MOMP) there is not a single optimal solution that simultaneously optimizes all the objective functions (Mavrotas, 2009; Trisna, 2016). Therefore, the decision makers have to choose a "most preferred" solution among the non-dominated solutions. A non-dominated, or Pareto optimal, solution is one that cannot be improved in one objective without worsening its performance in at least another of the aspects that are to be optimized. The set of nondominated solutions of a problem is called its "Pareto front".

The most widespread methods for dealing with multi-objective problems, and multi-criteria MSW is not the exception, are the weighting or weighted-sum method (see, e.g., Coutinho-Rodrigues et al., 2012, Rossit et al., 2015; Tralhão et al., 2010) and the $\varepsilon$-constraint method (see, e.g., Mavrotas et al., 2015). These methods need to run several times to find the set of non-dominated solutions and, therefore, to construct, the Pareto front. However, the weighting needs a larger number of runs since many of them end in repeated solutions. In addition to that, solutions located in a non-convex region of the Pareto front cannot be reached with this method (Caramia \& Dell'Olmo, 2008; Méndez et al., 2014). 
On the other hand, despite its advantages, the $\varepsilon$-constraint method has also some weaknesses that have been addressed in the literature (Mavrotas, 2009; Zhang \& Reimann, 2014). Among them we can cite the generation of dominated solutions. This wastes time and computational effort. Because of this problem, some authors have proposed modifications in order to overcome it, like in Zhang and Reimann (2014) or in Mavrotas (2009). The former method, called the augmented $\varepsilon$-constraint method (AUGMECON), which was later improved in Mavrotas and Florios (2013), is the one that we will use in this paper. Since it may not be still too familiar, we provide a short presentation of AUGMECON in subsection 2.2.1. For an exhaustive description, we refer to Mavrotas and Florios, 2013. In parallel we apply the weighting method to our problem in order to compare the performances of both methods. Subsection 2.2.2 introduces a common basis of comparison between both multi-objective techniques.

\subsubsection{The AUGMECON method}

The augmented $\varepsilon$-constraint method is a variation of the conventional $\varepsilon$-constraint method. It addresses some weaknesses of the $\varepsilon$-constraint one, namely, the guarantee of Pareto optimality of the solution both in the payoff table as well as in the generation process that produces unnecessarily long computing times. In the AUGMECON method, the problem to be solved has the formulation given by expressions (9)-(13).

$\max f_{1}(x)+e p s \cdot\left(a_{2} \frac{S_{2}}{r_{2}}+a_{3} \frac{S_{3}}{r_{3}}+\ldots+a_{n} \frac{S_{n}}{r_{n}}+\ldots+a_{N} \frac{S_{N}}{r_{N}}\right)$

subject to:

$\mathrm{f}_{2}(\mathrm{x})-\mathrm{S}_{2}=\varepsilon_{2}$

$\mathrm{f}_{3}(\mathrm{x})-\mathrm{S}_{3}=\varepsilon_{3}$

$f_{n}(x)-S_{n}=\varepsilon_{n}$

$x \in Z$

where $\varepsilon_{2}, \varepsilon_{3}, \ldots, \varepsilon_{n}$ are the parameters for the right hand side (RHS) for the specific iteration on the grid points of the objective functions $2,3, \ldots, n$. The parameters $r_{2}, r_{3}, \ldots, r_{n}$ are the ranges of the respective objective functions. $S_{2}, S_{3}, \ldots, S_{n}$ are the slack variables of the respective $\varepsilon$-constraints and eps is a constant value between $10^{-6}$ and $10^{-3}$.

In the original method (Mavrotas, 2009) the coefficients $a_{n}$ are all equal to one. Instead in AUGMECON2 (Mavrotas and Florios, 2013) the $a_{n}$ values are:

$a_{n}=10^{-(n-2)}, n=2, \ldots, N$

If there are alternative optima, this modification produces a lexicographic order to optimize on the objective functions. Then, the objective functions are optimized sequentially, i.e., first $f_{1}$, then $f_{2}$ and so on.

For each criterion, the range of the objective function is calculated as the difference between the best and the worst values of the payoff table. Then the range of the $k$-th objective function is divided in $q_{k}$ equal intervals using $q_{k}-1$ intermediate equidistant grid points. Thus, we obtain in total $q_{k}+1$ grid points 
that are used to systematically vary the right hand side of the restriction $\left(\varepsilon_{k}\right)$ of the $k$-th objective function. The total number of runs becomes $\left(q_{2}+1\right)\left(q_{3}+1\right) \ldots\left(q_{n}+1\right)$. If $r_{k}$ is the range of the objective function $k(k=2, \ldots, n)$, then the discretization step is:

step $_{\mathrm{k}}=\frac{\mathrm{r}_{\mathrm{k}}}{\mathrm{q}_{\mathrm{k}}}$

Then, the RHS of the corresponding constraint in the $t$-th iteration in the specific objective function is:

$\varepsilon_{\mathrm{kt}}=\mathrm{fmin}_{\mathrm{k}}+\mathrm{t} \cdot \mathrm{step}_{\mathrm{k}}$

Here $\operatorname{fmin}_{k}$ is the minimum taken from the payoff table and $t$ the counter for the specific objective function. At each iteration the surplus variable corresponding to the innermost objective function is checked. In our case, the objective function with $n=2$. Then we calculate the bypass integer coefficient as:

$\mathrm{b}=\left\lfloor\frac{\mathrm{S}_{\text {innermost }}}{\text { step }_{\text {innermost }}}\right\rfloor$.

When the surplus variable $S_{2}$ is larger than step $_{2}$, the same solution is obtained in the next iteration, with the only difference of a smaller surplus variable, which will change its value to $S_{2}-$ step $_{2}$. This makes the iteration redundant and, therefore, we can bypass it as no new Pareto optimal solution will be obtained in this run. The bypass coefficient $b$ actually indicates how many consecutive iterations we can jump ahead.

\subsubsection{The weighting method}

The weighting method uses the values of the payoff table to build the multi-objective function. Indeed, it summarizes the multi-objective nature of the problem in only one function by means of relative weights, measuring the distance between the current value of each objective and the ideal value of that criterion. Despite the existence of different ways to measure the distance from the optima, we implement a metric proposed by Zeleny (1974) which uses the best and nadir solutions of each objective in order to reduce the diverse criteria to a homogenous system of unit of measurement. Applying this metric to Eq. (1) we obtain (1').

$\operatorname{Min} w^{c}\left(\frac{f_{c^{*}}^{*}-f_{c}}{f_{c}^{*}-f_{c^{*}}}\right)+w^{d}\left(\frac{f_{d^{*}}^{*}-f_{d}}{f_{d^{*}}^{*}-f_{d^{*}}}\right)$

where $f_{c}^{*}$ and $f_{c^{*}}$ are the best and nadir points of the investment cost objective function; analogously, $f_{d}^{*}$ and $f_{d^{*}}$ are the best and nadir points of the average distance criteria; and $w^{c}$ and $w^{d}$ are the relative weights of the cost and the average distance objectives respectively. We assume that $w^{c}+w^{d}=1$ (Zeleny, 1974). In this method the grid points are defined selecting equally separated relative weights. If the number of grid points is $q$ we define the discretization step as:

step $=\frac{1}{q+1}$

Then, the relative weights for the $t$-th iteration are: 
$\mathrm{w}_{\mathrm{t}}^{\mathrm{c}}=\mathrm{t} \cdot \mathrm{step} \quad \mathrm{t}=1, \ldots, \mathrm{q}$

$\mathrm{w}_{\mathrm{t}}^{\mathrm{d}}=1-\mathrm{w}_{\mathrm{t}}^{\mathrm{c}}$

For the first iteration we use $w^{c}=$ step in order to avoid calculating the extreme point $w^{c}=0$ (and $\left.w^{d}=1\right)$. The opposite extreme weight is also avoided, by preventing the relative weights to adopt the values $w^{c}=1$ and $w^{d}=0$. Indeed, the maximum value for $w^{c}$ occurs when $t=q$ and is $\frac{q}{q+1}<1$.

\subsection{Implementation}

The daily per capita rate of waste generation in Bahía Blanca is $0.715 \mathrm{~kg}$ (PLAPIQUI CONICET, 2013), which is moderately less than the average rate of $0.77 \mathrm{~kg}$ calculated by Troschinetz and Mihelcic (2008) for developing countries. The composition of garbage in Bahía Blanca is $48 \%$ of organic waste, $18 \%$ of plastics, $11 \%$ of paper, $7 \%$ of glass and $16 \%$ of other materials. In order to separate the main kinds of garbage, we classify the waste in three flows: organic waste, plastics and other kinds of waste. These flows will have their corresponding bins.

We consider two different classes of bins: type 1 with a capacity of 1,000 liters and type 2 with a capacity of 2,000 liters. The maximum space in a collection site is $25 \mathrm{~m}^{2}$ and the space required by each container is $1 \mathrm{~m}^{2}$ for type 1 and $2 \mathrm{~m}^{2}$ for type 2 . The estimated density for organic waste, plastics and other kinds of garbage are 300, 65 and $150 \mathrm{~kg} / \mathrm{m}^{3}$ respectively (Henry, 1999). The maximum tolerated distance between a dwelling and its assigned collection site is $300 \mathrm{~m}$. The cost of opening a collection site is 50,000 monetary units (m.u.) and the cost of installing a bin are 3,000 m.u. and 5,000 m.u. for type 1 and 2 respectively. We consider three scenarios. The first one is based on 320 dwellings and 13 potential collection sites, distributed over an area of $160,000 \mathrm{~m}^{2}$. Scenario number two considers 720 dwellings and 18 potential collection sites, scattered over an area of $360,000 \mathrm{~m}^{2}$. The third scenario has 2,304 generators and 25 potential collection sites, distributed over an urban area of $640,000 \mathrm{~m}^{2}$. A dwelling can be a large, medium or small garbage generator according to a custom probabilistic distribution that represents a crowded urban area. The number of equivalent people that corresponds to each kind of generator is based on the average number of people per housing unit in the city of Bahía Blanca, which is approximately three people (value taken from the Statistics' Department of the Province of Buenos Aires, Argentina). A large generator is simulated as a building with thirteen housing units. Two housing units or a large retail prperty constitute a medium generator and a small generator consists of a single housing unit or a small shop.

The software used to solve the problem is CPLEX 12.5.1 and the problem was modeled in GAMS 24.1.3. The computer used has the following characteristics: processor Intel Core I5-4210U, CPU @ $2.7 \mathrm{GHz}$, RAM memory of $3.5 \mathrm{~GB}$ and an operating system of 64 bits. The payoff tableau was calculated using the lexicographic method. The solver time limit for calculating the values for the payoff table was 3,000 seconds. After that, we applied the two multi-objective methods, the weightedsum and AUGMECON2, in order to solve the model (1)-(8) and obtain a set of non-dominated solutions. Initially we gave ten grid points to the solver. In a second instance, the number of grid points was doubled. These grid points are uniformly separated. In the case of the AUGMECON2 this separation correspond to the range of the investment cost objective (refer to subsection 2.2.1). In the weighting method, instead, the uniformity relies on equally separated relatives weights (see subsection 2.2.2). In both models, the time limit for finding a multi-objective optimal solution in each run is 2,000 seconds.

For each scenario, we recorded the number of efficient solutions obtained, the computing time in seconds and the average deviation from the optima. The metric we used to estimate this deviation is the 
relative gap, which is calculated by the MIP (mixed-integer programming) module of CPLEX according to the following equation:

Relative gap $=\frac{|\mathrm{BP}|-|\mathrm{BF}|}{1^{-10}+|\mathrm{BF}|}$,

where $B F$ is the value of the objective function at the current best integer solution found so far and $B P$ is the value of the objective function at the best possible integer solution (see the manual of this solver ${ }^{1}$ ).

In the case of the weighting method, we calculated the average of the relative gaps considering only the deviations associated with non-inferior and unrepeated solutions. Indeed, the inferior solutions (generated because of time limit) were simply discarded and in the case of repeated solutions (more than one combination of weights leading to to the same efficient solution) we chose the one with the smaller relative gap and dismissed the others. In the case of AUGMECON2, there is no need of these special considerations because by design it does not produce inferior or repeated solutions.

\section{Results}

The results obtained for the first scenario are presented in Table 1 (ten grid points) and Table 2 (twenty grid points). For the second scenario, the outcomes are shown in Table 3 (ten grid points) and Table 4 (twenty grid points). Finally, Table 5 (ten grid points) and Table 6 (twenty grid points) show the results of the third scenario.

Table 1

Results for the first scenario and ten grid points

\begin{tabular}{llll}
\hline Multi-objective method & $\begin{array}{l}\text { Number of efficient } \\
\text { solutions }\end{array}$ & $\begin{array}{l}\text { Computing time } \\
\text { (seconds) }\end{array}$ & $\begin{array}{l}\text { Average } \\
\text { deviation from the optima }\end{array}$ \\
\hline Weighted-sum & 10 & 364.89 & 0.000000 \\
AUGMECON2 & 10 & 1196.27 & 0.000000 \\
\hline
\end{tabular}

\section{Table 2}

Results for the first scenario and twenty grid points.

\begin{tabular}{llll}
\hline Multi-objective method & $\begin{array}{l}\text { Number of efficient } \\
\text { solutions }\end{array}$ & $\begin{array}{l}\text { Computing time } \\
\text { (seconds) }\end{array}$ & $\begin{array}{l}\text { Average } \\
\text { deviation from the optima }\end{array}$ \\
\hline Weighted-sum & 8 & 1431.75 & 0.000000 \\
AUGMECON2 & 17 & 1488.30 & 0.000000 \\
\hline
\end{tabular}

Table 3

Results for the second scenario and ten grid points.

\begin{tabular}{llll}
\hline Multi-objective method & $\begin{array}{l}\text { Number of efficient } \\
\text { solutions }\end{array}$ & $\begin{array}{l}\text { Computing time } \\
\text { (seconds) }\end{array}$ & $\begin{array}{l}\text { Average } \\
\text { deviation from the optima }\end{array}$ \\
\hline Weighted-sum & 7 & 11239.87 & 0.002067 \\
AUGMECON2 & 10 & 8285.61 & 0.001621 \\
\hline
\end{tabular}

Table 4

Results for the second scenario and twenty grid points.

\begin{tabular}{llll}
\hline Multi-objective method & $\begin{array}{l}\text { Number of efficient } \\
\text { solutions }\end{array}$ & $\begin{array}{l}\text { Computing time } \\
\text { (seconds) }\end{array}$ & $\begin{array}{l}\text { Average } \\
\text { deviation from the optima }\end{array}$ \\
\hline Weighted-sum & 12 & 26892.59 & 0.005458 \\
AUGMECON2 & 18 & 24408.58 & 0.012701 \\
\hline
\end{tabular}


Table 5

Results for the third scenario and ten grid points.

\begin{tabular}{llll}
\hline Multi-objective method & $\begin{array}{l}\text { Number of efficient } \\
\text { solutions }\end{array}$ & $\begin{array}{l}\text { Computing time } \\
\text { (seconds) }\end{array}$ & $\begin{array}{l}\text { Average } \\
\text { deviation from the optima }\end{array}$ \\
\hline Weighted-sum & 9 & 17256.55 & 0.025371 \\
AUGMECON2 & 10 & 16873.17 & 0.016303 \\
\hline
\end{tabular}

Table 6

Results for third scenario and twenty grid points.

\begin{tabular}{llll}
\hline Multi-objective method & $\begin{array}{l}\text { Number of efficient } \\
\text { solutions }\end{array}$ & $\begin{array}{l}\text { Computing time } \\
\text { (seconds) }\end{array}$ & $\begin{array}{l}\text { Average } \\
\text { deviation from the optima }\end{array}$ \\
\hline Weighted-sum & 14 & 36022.58 & 0.014260 \\
AUGMECON2 & 15 & 29893.08 & 0.020692 \\
\hline
\end{tabular}

To visualize the results we depict the Pareto fronts of the outcomes with twenty grid points. Fig. 1 and 2 show the results for the first scenario solved with the weighting and the AUGMECON2 methods respectively. For the second (Fig. 3 and 4) and third scenario (Fig. 5 and 6), the same order of presentation is used. As we have done to calculate the average gap, we discarded the inferior and repeated solutions of the weighting method. The Pareto front was approximated by joining the efficient solutions (represented by white dots) with straight segments.

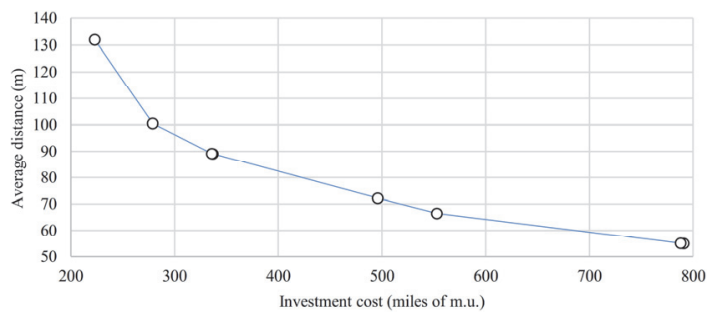

Fig. 1. Pareto front of the first scenario. Number of grid points $=20$. Multi-objective approach $=$ weighted-sum

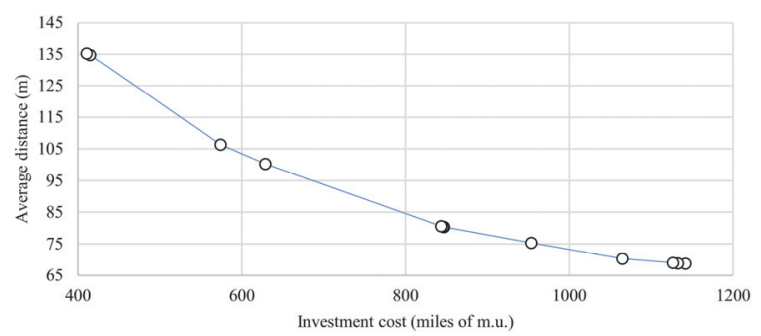

Fig. 3. Pareto front of the second scenario. Number of grid points $=20$. Multi-objective approach $=$ weighted-sum

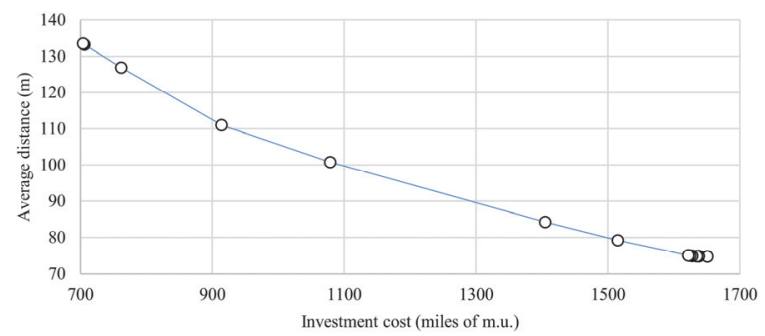

Fig. 5. Pareto front of the third scenario. Number of grid points $=20$. Multi-objective approach $=$ weighted-sum

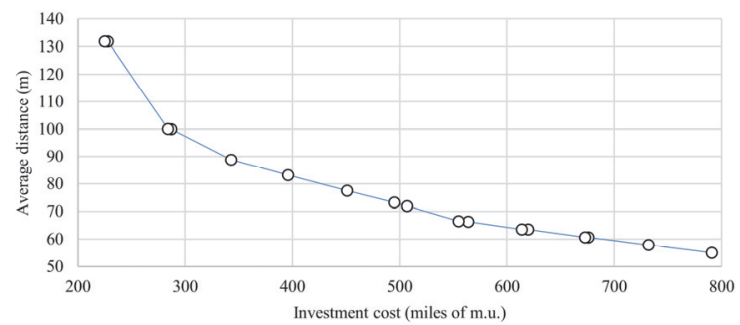

Fig. 2. Pareto front of the first scenario. Number of grid points $=20$. Multi-objective approach $=$ AUGMECON2

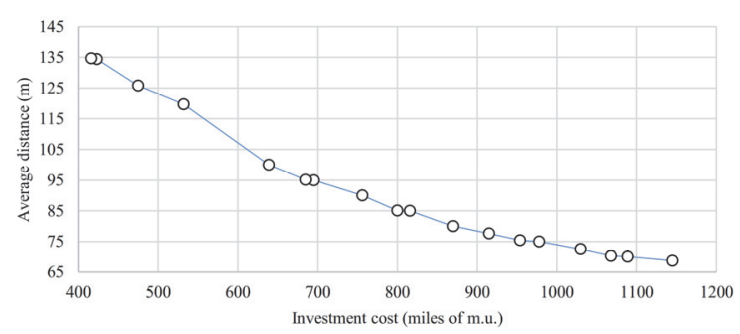

Fig. 4. Pareto front of the second scenario. Number of grid points $=20$. Multi-objective approach $=$ AUGMECON2

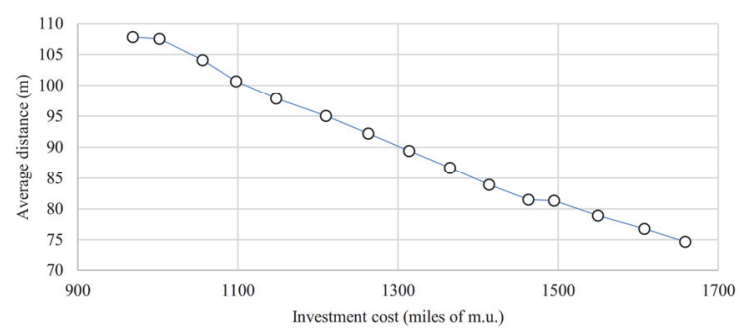

Fig. 6. Pareto front of the third scenario. Number of grid points $=20$. Multi-objective approach $=$ AUGMECON2 
In the ten grid points run of the first scenario (Table 1), both methods found ten non-dominated solutions with no deviation from the optima. However, the weighting method required a smaller amount of time. In the twenty grid points run (Table 2), the situation changes. The number of non-inferior solutions of the traditional approach is eight, against seventeen of AUGMECON2. Although the weighted-sum still demands a shorter computing time, the difference between the approaches is smaller. The average deviation is again zero. This null relative gap may be due to the simplicity of the scenario (this is the smallest scenario of the three), which allows the solver to find the optimal values within the time limit of 2,000 seconds independently of the multi-objective approach applied.

In the second scenario, the weighting method finds less efficient solutions in both runs (Table 3 and Table 4). This difference is bigger for the run on twenty grid points. In addition to that, the weighting method requires more computing time in both runs. However, the classical approach obtains a smaller deviation gap for the larger run. In the ten grid points run the relation between both relative gaps is the other way around.

In the last scenario, the results are unanimously better under the AUGMECON2 method. Both the computing time and the number of Pareto optimal solutions (Table 5 and Table 6) improve over those of the weighting method. The situation with the relative gap repeats the pattern of the second scenario, where in the short run AUGMECON2 performs better than the weighting method (Table 5) and worse in the long run (Table 6).

The other aspect to be compared is the distribution of solutions along the Pareto front. For the sake of brevity we only present the plots of the outcome of runs with twenty grid points, which ensures a richer visualization of Pareto fronts. The comparisons in the three scenarios involve, respectively, Fig. 1 vs. Fig. 2, Fig. 3 vs. Fig. 4 and Fig. 5 vs. Fig. 6 . We can see that not only the AUGMECON2 produces a denser curve due to a larger number of efficient solutions, but also generates a more regular distribution of the solutions along the front. This feature can be attributed to the use of separated grid points by this method.

In contrast, when we applied equally separated relative weights in the weighting method, the results were rather different, obtaining not only a more heterogeneous distribution of solutions but also repetitions. In some extreme situations, we obtained for a relatively large range of different weights the same solution. This is related to the fact that in the weighted-sum, a uniform spread of relative weights, in general, does not produce a uniform spread of points on the Pareto curve (Caramia and Dell'Olmo, 2008). Indeed, Fig. 1, 3 and 5 show that efficient solutions are concentrated on certain parts of the Pareto front, while significant portions of the trade-off curve do not include any efficient solution. In the three fronts, there is an especially high concentration of solutions in the zone of larger investment costs.

\subsection{Discussion of results}

In regards to the resolution algorithm, the AUGMECON2 method has in general shown a better performance than the weighted-sum on the scenarios analyzed in this paper. The strong points of the AUGMECON2 that have been observed in this paper, and have already been recognized in the literature, are: shorter computing times, larger number of efficient solutions and the possibility of moderate control on the distribution of efficient points along the front. These beneficial results can be attributed to the capacity of this method to exploit the information stored in the $\varepsilon$-constraint slack variables and, therefore, avoiding runs leading to repeated solutions. Conversely, the weighting approach, which lacks this feature, calculates a solution for every grid point, without yielding a repeated solution or, due to the finite computation time, a dominated solution. Despite all these characteristics in favor of the AUGMECON2 method, we must mention some exceptions. For example, in the ten grid points runs of the smaller scenario, the weighting method provided the same number of Pareto optimal 
solutions at a shorter computation time. Moreover, with respect to the average deviation from the optima, the results are not categorical. The weighted-sum method, in general, has a better performance when more solutions are demanded. Nevertheless, we should note that the average deviation from the optima is computed only with the non-inferior and unrepeated solutions of the weighting method. AUGMECON2 is more effective and does not generate repeated or inferior solutions, without the need of making similar special considerations. The regular distribution of the points in the Pareto front is a key point since it allows MSW decision-maker to have a more comprehensive view of the problem.

As a summary, besides exhibiting the strong points of the AUGMECON2 in comparison to the weighting method, we think that this work shows the suitability of the proposed model for improving the efficiency of the MSW of a developing country's medium size city. In future work, we would like to perform more test on both larger simulations and real-life data.

\section{Conclusions}

It is a well-known fact that waste handling in many developing countries is far from being efficient. This situation applies to Argentina where, in general, the recycling rates are at the lowest levels. We consider that source separation is a crucial activity that facilitates the recycling and reutilization of waste products. In the case of study, the southern city of Bahía Blanca, where the landfill, with its capacity near to collapse, is the receptor of almost all the produced garbage, policies encouraging recycling are of foremost importance. However, this situation is replicated in other developing countries' cities, that struggle to find a path of sustainability in the management of MSW. Contarary to the case of metropolises, in many of the medium size cities of these countries, the current management of MSW is mainly based on experience and empiric knowledge of decision-makers. Even though experience is a priceless asset for dealing with this problem, the contribution that Operations Research approaches can make to improvement of MSW systems should not be ignored. The evidence found in the literature of the benefits of applying quantitative models for improving MSW systems is overwhelming. This is why in this paper we have proposed a multi-objective integer model to optimize the location of collection sites in an urban area and allow source separation. If implemented, this would mean the replacement of the unsorted door-to-door system by a source separation community bins system. Moreover, this new system would simplify routing schedules since less places have to be visited. We have tested the model on simulated instances of the urban structure of Bahía Blanca seeking minimal investment costs and minimal average distance between dwellings and the assigned sites. We have solved the model with two different approaches: the augmented $\varepsilon$-constraint method and the weighting method. The model allows us to obtain the Pareto front of the problem. Even though the instances correspond to Bahía Blanca, we consider that this model can be applied to other cities with similar characteristics.

\section{References}

Badran, M. F., \& El-Haggar, S. M. (2006). Optimization of municipal solid waste management in Port Said-Egypt. Waste Management, 26(5), 534-545.

Benes, E., \& Milmanda, B. F. (2012). El nuevo liderazgo sindical en la Argentina postneoliberal: el caso del gremio de camioneros. Desarrollo Económico: Revista de Ciencias Sociales, 52(205), 3162.

Bonomo, F., Durán, G., Larumbe, F., \& Marenco, J. (2012). A method for optimizing waste collection using mathematical programming: a Buenos Aires case study. Waste Management \& Research, 30(3), 311-324.

Broz, D., Rossit, D. A., Rossit, D. G., \& Cavallin, A. (2016). Challenge in supply chains management in Argentina: The case of forestry-industrial sector. In Proceedings of the 8th International Conference on Production Research ICPR-AMERICAS'2016. Valparaíso, Chile. 
Caramia, M., \& Dell' Olmo, P. (2008). Multi-objective optimization. In their Multi-objective Management in Freight Logistics: Increasing Capacity, Service Level and Safety with Optimization Algorithms, Springer Science \& Business Media, Berlin, 11-36.

Crespo, F. A., \& Paredes, R. D. (2009). A mechanism for efficient and fair NIMBY location. DYNA, 76(160), 293-303.

Coutinho-Rodrigues, J., Tralhão, L., \& Alçada-Almeida, L. (2012). A bi-objective modeling approach applied to an urban semi-desirable facility location problem. European Journal of Operational Research, 223(1), 203-213.

Di Felice, P. (2014a). Integration of spatial and descriptive information to solve the urban waste accumulation problem. Procedia-Social and Behavioral Sciences, 147, 182-188.

Di Felice, P. (2014b). Integration of spatial and descriptive information to solve the urban waste accumulation problem: A pilot study. Procedia-Social and Behavioral Sciences, 147, 592-597.

Erkut, E., Karagiannidis, A., Perkoulidis, G., \& Tjandra, S. A. (2008). A multicriteria facility location model for municipal solid waste management in North Greece. European Journal of Operational Research, 187(3), 1402-1421.

Ferri, G. L., Chaves, G. D. L. D., \& Ribeiro, G. M. (2015). Analysis and location of urban solid waste collection/inspection centers for a reverse logistics network: a case study in São Mateus-ES. Production, 25(1), 27-42.

Gandara, M. P., \& Guerrero, E. M. (2013). Indicadores ambientales para la gestión de los residuos sólidos domiciliarios en Rosario, Argentina. DELOS, 6(16).

Ghiani, G., Laganà, D., Manni, E., Musmanno, R., \& Vigo, D. (2014a). Operations research in solid waste management: A survey of strategic and tactical issues. Computers \& Operations Research, 44, 22-32.

Ghiani, G., Manni, A., Manni, E., \& Toraldo, M. (2014b). The impact of an efficient collection sites location on the zoning phase in municipal solid waste management. Waste Management, 34(11), 1949-1956.

Ghiani, G., Laganà, D., Manni, E., \& Triki, C. (2012). Capacitated location of collection sites in an urban waste management system. Waste management, 32(7), 1291-1296.

Henry J. G. (1999). Residuos Sólidos. In Henry J. G. \& Heinke G. W. (Eds) Ingeniería Ambiental, Pearson Educación, México, pp 567-619.

Hoornweg, D., \& Bhada-Tata, P. (2012). What a waste: a global review of solid waste management. Urban Development Series Knowledge Papers, 15, 1-98.

Hui, Y., Li'ao, W., Fenwei, S., \& Gang, H. (2006). Urban solid waste management in Chongqing: Challenges and opportunities. Waste management, 26(9), 1052-1062.

Hwang, C. L., \& Masud, A. S. M. (2012). Multiple objective decision-making methods and applications: a state-of-the-art survey (Vol. 164). Berlin: Springer Science \& Business Media.

Mavrotas, G. (2009). Effective implementation of the $\varepsilon$-constraint method in multi-objective mathematical programming problems. Applied Mathematics and Computation, 213(2), 455-465.

Mavrotas, G., \& Florios, K. (2013). An improved version of the augmented $\varepsilon$-constraint method (AUGMECON2) for finding the exact pareto set in multi-objective integer programming problems. Applied Mathematics and Computation, 219(18), 9652-9669.

Mavrotas, G., Gakis, N., Skoulaxinou, S., Katsouros, V., \& Georgopoulou, E. (2015). Municipal solid waste management and energy production: Consideration of external cost through multi-objective optimization and its effect on waste-to-energy solutions. Renewable and Sustainable Energy Reviews, 51, 1205-1222.

Méndez M., Álamo A., Frutos M., \& Aguasca R. (2014). Métodos. Agregación. In their Fundamentos de ayuda multicriterio a la decisión, CEANI, Las Palmas de Gran Canaria, pp 24-33.

Ministry of Environment and Sustainable Development of Argentina, MADS (2016). "Mapas Críticos Gestión de Residuos Marzo 2016". Retrieved from: http://observatoriorsu.ambiente.gob.ar/estadisticas [online, date consulted: 6th March 2017].

Parrot, L., Sotamenou, J., \& Dia, B. K. (2009). Municipal solid waste management in Africa: Strategies and livelihoods in Yaoundé, Cameroon. Waste management, 29(2), 986-995. 
Pérez-Salazar, M. D. R., Mateo-Díaz, N. F., García-Rodríguez, R., Mar-Orozco, C. E., \& Cruz-Rivero, L. (2015). A genetic algorithm to solve a three-echelon capacitated location problem for a distribution center within a solid waste management system in the northern region of Veracruz, Mexico. DYNA, 82(191), 51-57.

Poletto, M., De Mori, P. R., Schneider, V. E., \& Zattera, A. J. (2016). Urban solid waste management in Caxias do Sul/Brazil: practices and challenges. Journal of Urban and Environmental Engineering, 10(1), 50-56.

Pradhan, P. K., Mohanty, C. R., Swar, A. K., \& Mohapatra, P. (2012). Urban solid waste management of Guwahati city in north-east India. Journal of Urban and Environmental Engineering, 6(2), 6773.

PLAPIQUI UNS-CONICET (2013). Análisis Estadístico de los Residuos Sólidos Domiciliarios de Bahía Blanca.

Rossit, D. G., Broz, D., Rossit, D. A., Frutos, M. and Tohmé, F. (2015), Una herramienta logística para la localización de contenedores de residuos separados en origen. In Mazzeo, N. M. \& Muzlera Klappenbach, A. (Eds) Avances en Gestión Integral de Residuos Sólidos Urbanos 2014-2015, INTI, Buenos Aires, pp.50-69.

Salemi, H. (2016). A hybrid algorithm for stochastic single-source capacitated facility location problem with service level requirements. International Journal of Industrial Engineering Computations, 7(2), 295-308.

Tralhão, L., Coutinho-Rodrigues, J., \& Alçada-Almeida, L. (2010). A multiobjective modeling approach to locate multi-compartment containers for urban-sorted waste. Waste Management, 30(12), 2418-2429.

Trisna, T., Marimin, M., Arkeman, Y., \& Sunarti, T. (2016). Multi-objective optimization for supply chain management problem: A literature review. Decision Science Letters, 5(2), 283-316.

Troschinetz, A. M., \& Mihelcic, J. R. (2009). Sustainable recycling of municipal solid waste in developing countries. Waste Management, 29(2), 915-923.

Tufró, V., Chalde, J. I., Campos, R. I., \& Pérez Toscani, M. (2012). A local model for a global problem. The case of the city of Salta, Argentina. In Proceedings of the 2012 International Solid Waste Association Congress. Florence, Italy.

Toso, E. A. V., \& Alem, D. (2014). Effective location models for sorting recyclables in public management. European Journal of Operational Research, 234(3), 839-860.

Zelany, M. (1974). A concept of compromise solutions and the method of the displaced ideal. Computers \& Operations Research, 1(3), 479-496.

Zhang, W., \& Reimann, M. (2014). A simple augmented $\varepsilon$-constraint method for multi-objective mathematical integer programming problems. European Journal of Operational Research, 234(1), $15-24$.

Zhuang, Y., Wu, S. W., Wang, Y. L., Wu, W. X., \& Chen, Y. X. (2008). Source separation of household waste: a case study in China. Waste Management, 28(10), 2022-2030.

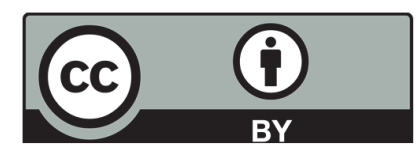

(C) 2017 by the authors; licensee Growing Science, Canada. This is an open access article distributed under the terms and conditions of the Creative Commons Attribution (CC-BY) license (http://creativecommons.org/licenses/by/4.0/). 